Im Wasserstoffbrückenassoziat des Fluorenons in Hexafluorisopropanol tritt eine weitere neue Bande bei $31500 \mathrm{~cm}^{-1}$ auf, die etwa die gleiche Intensität wie die Bande bei $31200 \mathrm{~cm}^{-1}$ hat. Diese Bande dürfte als eine Schwingungsbande des ungestört verbotenen Übergangs zu deuten sein. Im nichtwasserstoffverbrückten Fluorenon wird diese Bande durch die benachbarte Elektronenbande überlagert; nur im glasig eingefrorenen Zustand im polaren Lösungsmittel Di-n-propyläther-Isopentan (4:1) kann die Bande bei etwa $32100 \mathrm{~cm}^{-1}$ als Schulter der Bande bei $32200 \mathrm{~cm}^{-1}$ erkannt werden (vgl. Abb. 1).

In Lösungen von Fluorenon in Hexafluorisopropanol macht sich noch eine weitere, weniger intensive Bande bei etwa $28900 \mathrm{~cm}^{-1}$ bemerkbar, die nicht einfach einzuordnen ist. Möglicherweise wird diese Bande durch ein höheres Wasserstoffbrücken- assoziat verursacht, das erst bei sehr hoher Hexafluorisopropanolkonzentration in kleinerer Konzentration vorliegt.

Die Konzentrationsabhängigkeit der Spektren bei kleineren Konzentrationen $\left(c_{\text {Fluorenon }} \approx 10^{-2} \mathrm{~mol} / 1\right.$, $c_{\text {Hexafluorisopropanol }} \approx 10^{-2}$ bis $1 \mathrm{~mol} / 1$ ) ermöglicht eine Bestimmung der Gleichgewichtskonstante $25 K_{\mathrm{c}}=(8,1 \pm 1,2) 1 / \mathrm{mol}$. Der nach der gleichen Methode bestimmte Extinktionskoeffizient des Wasserstoffbrückenassoziats ist innerhalb der Fehler nahezu mit dem in Abb. 7 dargestellten Spektrum in $100 \%$ Hexafluorisopropanol identisch.

Unser aufrichtiger Dank gilt Herrn Professor Dr. G. BrIEgleb für sein stetes Interesse und dem Fonds der Chemischen Industrie und der Deutschen Forschungsgemeinschaft für die Unterstützung dieser Untersuchungen. Herrn Dr. Middleton danken wir für die U̇berlassung des Hexafluorisopropanols.

25 W. Liptay, Ber. Bunsenges. physik. Chem. 65, 375 [1961].

\title{
Untersuchungen zum Temperaturgang der F-Absorptionsbande
}

\author{
Lore Kern-Bausch
}

\author{
Sektion Physik der Universität München
}

(Z. Naturforsch. 23 a, 393-400 [1968]; eingegangen am 9. November 1967)

\begin{abstract}
Wir behandeln die F-Zentren-Absorption für zwei verschiedene Modelle im Rahmen der adiabatischen Näherung. Zunächst wird die gesamte F-Bande mit der Temperatur als Parameter bestimmt, und damit das Maximum für den Fall, daß zwei Oszillatoren gleicher Frequenz mit dem Übergang des F-Zentren-Elektrons in einen angeregten Zustand korreliert sind; dabei berücksichtigt man Terme, die durch eine Abweichung vom Franck-Condon-Prinzip bedingt werden. In $\S 2$ berechnen wir die Absorptionskurve für ein Modell, bei dem sich die angekoppelten Gitterschwingungen beim Elektronenübergang ändern; allerdings sind jetzt die Ubergänge gemäß der Franck-Condon-Approximation eingeschränkt. Es zeigt sich, daß man den Temperaturgang des Maximums der Bande weder mit dem ersten Modell, das auf einem Vorschlag von $W_{\text {AGNER }}$ beruht, noch durch Änderung der Oszillatorfrequenzen, wie die temperaturabhängige Lageverschiebung z. B. auch von Dexter und PRYCE dargestellt wird, erklären kann. Im letzten Abschnitt gehen wir auf die Ergebnisse der eben genannten Arbeiten im Zusammenhang mit unseren Rechnungen ein.
\end{abstract}

Die experimentellen Untersuchungen der Absorptionsbande von F-Zentren in Alkalihalogeniden aus dem Jahre 1933 von Molswo ${ }^{1}$ ergaben deutlich, daß sich das Maximum der Bande mit steigender Temperatur nach der Seite geringerer Energie hin verschiebt. Wie ein Vergleich mit neuesten Daten ${ }^{2}$ zeigt, konnte schon MoLlwo sowohl die Richtung des

1 E. Mollwo, Z. Phys. 85, 62 [1933].

2 H. PIck, Springer Tracts in Modern Physics 38, SpringerVerlag Berlin-Heidelberg-New York 1965; - Jordan J. Markham, F-Centers in Alkali Halides, Solid State Phys. Suppl. 1966, 8.
Temperaturganges als auch seine Größenordnung von $0,1 \mathrm{eV}$ zwischen $0{ }^{\circ} \mathrm{K}$ und $300{ }^{\circ} \mathrm{K}$ richtig angeben.

SmakUla ${ }^{3}$ hat als erster eine Theorie für Absorptionsbanden im Festkörper entworfen. Im Anschluß daran wurde, wie man aus zwei Übersichtsartikeln von MARKhaM ${ }^{4}$ entnehmen kann, verschiedentlich

3 A. Smakula, Z. Phys. 59, 603 [1930].

4 J. J. Markham, Rev. Mod. Phys. 31, 956 [1959] ; - s. Anmerkung ${ }^{2}$. 
versucht, eine Erklärung für die Temperaturabhängigkeit des Maximums zu finden. Marкham weist an mehreren Stellen seines Buches ${ }^{5}$ jedoch darauf hin, $\mathrm{da}$ ß solche Versuche bis heute noch zu keiner befriedigenden Lösung geführt haben. Er geht aber nicht auf Arbeiten von $\mathrm{W}_{\mathrm{AGNER}}{ }^{6}$ ein, der im Rahmen seiner Abhandlungen zur F-Zentren-Theorie eine Deutung der Temperaturverschiebung erwartet, wenn man eine Entwicklung bis zu Gliedern erster Ordnung der Variationsparameter der Elektronenwellenfunktionen nach den Normalkoordinaten der ankoppelnden Gitteroszillatoren ansetzt. Auch $\mathrm{PICK}^{7}$ erhofft auf Grund der WaGNerschen Vorstellungen eine Klärung des Temperatureffekts.

Da W anschreibt, sie aber in den weiteren Rechnungen ohne Angabe von Abschätzungen vernachlässigt, werden wir hier zunächst das W $_{\text {AGNersche Modell }}$ numerisch untersuchen. In $\S 2$ wird dann ein Modell behandelt, bei dem die mitwirkenden Oszillatoren im Grund- und im angeregten Zustand verschiedene Frequenzen haben.

\section{$\S 1$. Numerische Berechnung der F-Absorptions- bande in $\mathrm{KCl}$ nach Wagner}

Eine einfallende Lichtwelle erzeuge im Innern eines mit F-Zentren durchsetzten Kristalls ein elektrisches Feld vom Betrag $E(t)$; dann gilt für die gesamte Wahrscheinlichkeit im Zeitraum $T$ eines optischen Übergangs des uns interessierenden Systems, nämlich Störstelle mit lokalisiertem Elektron, von einem Zustand $v$ in einen Zustand $v^{\prime}$.

$$
P_{v v^{\prime}}=\frac{4 \pi^{2}}{\hbar^{2}}\left|E\left(\omega_{v v^{\prime}}\right)\right|^{2}\left|\mathfrak{e} \cdot D_{v v^{\prime}}\right|^{2} ;
$$

$E(\omega)$ ist die Spektralfunktion von $E(t)$ und $D_{v v^{\prime}}$ das Übergangsmatrixelement, auf das wir zunächst eingehen wollen.

Wir betrachten den Übergang $n, m_{1}, m_{2}, m_{3}, \ldots$ $\rightarrow n^{\prime}, m_{1}{ }^{\prime}, m_{2}{ }^{\prime}, m_{3}{ }^{\prime}, \ldots$; dabei zerlegen wir die oben in $v$ und $v^{\prime}$ zusammengefaßten Quantenzahlen zweier Zustände des Systems in $m_{i}$ und $m_{i}{ }^{\prime}$, die die Quantenzahlen der am Übergang beteiligten Oszillatoren charakterisieren, und in $n$ und $n^{\prime}$, die die Elektronenzustände kennzeichnen.

Unter der Annahme, daß bei einem Übergang zwischen 2 Elektronenzuständen auch nur 2 Gitteroszillatoren eine Nullpunktsverschiebung erleiden ${ }^{8}$ und daß sich die Gesamtwellenfunktion als Produkt aus Elektronenfunktion $\psi$ und Oszillatorfunktionen $\varphi$ darstellt, gilt für das Dipolmatrixelement ${ }^{9}$

$$
\begin{gathered}
D_{n m_{1} m_{2} ; n^{\prime} m_{1} m_{2}{ }^{\prime}}=-e \int \psi_{n}{ }^{*}\left(\mathfrak{r}, q_{1}{ }^{(n)}, q_{2}{ }^{(n)}\right) \mathfrak{r} \psi_{n^{\prime}}\left(\mathfrak{r}, q_{1}{ }^{\left(n^{\prime}\right)}, q_{2}{ }^{\left(n^{\prime}\right)}\right) \varphi_{m_{1}}^{*}\left(q_{1}{ }^{(n)}\right) \varphi_{m_{2}}^{*}\left(q_{2}{ }^{(n)}\right) \\
\cdot \varphi_{m_{1}{ }^{\prime}}\left(q_{1}{ }^{\left(n^{\prime}\right)}\right) \varphi_{m_{2}}\left(q_{2}{ }^{\left(n^{\prime}\right)}\right) \mathrm{d}^{3} \mathfrak{r} \mathrm{d} q_{1}{ }^{(n)} \mathrm{d} q_{2}{ }^{(n)} ;
\end{gathered}
$$

$\mathfrak{r}$ ist der Ortsvektor im Koordinatenraum des Störstellenelektrons, $q_{i}^{(n)}$ und $q_{i}^{\left(n^{\prime}\right)}$ sind die Normalkoordinaten der Oszillatoren im Elektronenzustand $n$ und $n^{\prime}$, wobei

$$
q_{i}^{\left(n^{\prime}\right)}=q_{i}^{(n)}+a_{i}^{\left(n n^{\prime}\right)} .
$$

Die $a_{i}{ }^{\left(n n^{\prime}\right)}$ beschreiben die Verschiebungen aus den Oszillatorruhelagen im angeregten Zustand ${ }^{10}$. In Gl. (2) wurde die Abhängigkeit der $\varphi$ von den Eigenfrequenzen der Oszillatoren nicht mitangegeben, da wir annehmen wollen, daß beide Oszillatoren eine gleiche und beim Übergang gleichbleibende Eigenfrequenz $\omega_{0}$ haben, die wir durch die Grenzfrequenz der longitudinal-optischen Schwingungen approximieren $^{11}$.

5 Siehe Anm. ${ }^{2}$.

${ }^{6}$ M. W AGNER, Z. Naturforsch. 15 a, 889 [1960]; 16 a, 302 [1961]. (Diese Arbeiten sollen im folgenden mit (I) bzw. (II) bezeichnet werden.)

7 Siehe Anm. 2, S. 70.
Wir beschränken uns auf die Berechnung der Hauptbande des F-Zentrums, die beim Übergang $1 s-2 p$ genaugenommen durch Überlagerung dreier Banden (3 verschiedene 2p-Funktionen) entsteht. Mit den Wasserstoffunktionen

$$
\begin{gathered}
\psi_{1 \mathrm{~s}}=\pi^{-1 / 2}\left(\alpha^{(1 \mathrm{~s})}\right)^{3 / 2} \exp \left\{-\alpha^{(1 \mathrm{~s})} r\right\} \quad \text { und } \\
\psi_{2 \mathrm{p}}=\pi^{-3 / 2}\left(\alpha^{(2 \mathrm{p})}\right)^{5 / 2} r \exp \left\{-\alpha^{(2 \mathrm{p})} r\right\} \cos \vartheta
\end{gathered}
$$

erhält man für das Elektronenintegral

$$
\begin{aligned}
\int \psi_{1 \mathrm{~s}}^{*}\left(\mathfrak{r}, \alpha^{(1 \mathrm{~s})}\right) \mathfrak{r} \psi_{2 \mathrm{p}}\left(\mathfrak{r}, \alpha^{(2 \mathrm{p})}\right) \mathrm{d}^{3} \mathfrak{r} \\
=\{0,0,1\} 32 \frac{\left(\alpha^{(1 \mathrm{~s})}\right)^{3 / 2}\left(\alpha^{(2 \mathrm{p})}\right)^{\mathrm{s} / \mathrm{s}}}{\left(\alpha^{(1 \mathrm{~s})}+\alpha^{(2 \mathrm{p})}\right)^{5}} .
\end{aligned}
$$

\footnotetext{
${ }^{8}$ Siehe (I) $\$ 7$.

9 Siehe (II) $\$ 1$.

10 Vgl. (I) § 7; man beachte dort die andere Vorzeichenwahl!

11 Für KCl: $\omega_{0}=3,985 \cdot 10^{13} \mathrm{~s}^{-1}$.
} 
Wie WAGNER machen wir für die Variationsparameter der Wellenfunktionen folgenden Ansatz:

$$
\begin{aligned}
& \alpha^{(1 \mathrm{~s})}=\alpha_{0}^{(1 \mathrm{~s})}+\beta_{1}^{(1 \mathrm{~s})} q_{1}^{(1 \mathrm{~s})}+\beta_{2}^{(1 \mathrm{~s})} q_{2}^{(1 \mathrm{~s})} ; \\
& \alpha^{(2 \mathrm{p})}=\alpha_{0}^{(2 \mathrm{p})}+\beta_{1}^{(2 \mathrm{p})} q_{1}^{(2 \mathrm{p})}+\beta_{2}^{(2 \mathrm{p})} q_{2}^{(2 \mathrm{p})} .
\end{aligned}
$$

Bezeichnet $\alpha_{1 \mathrm{~s}}^{(2 \mathrm{p})}$ den Parameter $\alpha^{(2 \mathrm{p})}$ für die Ruhelagen $q_{1}^{(1 \mathrm{~s})}=q_{2}^{(1 \mathrm{~s})}=0$, so ergibt sich nach einigen Umformungen:

$$
\alpha^{(2 p)}=\alpha_{1 \mathrm{~s}}^{(2 \mathrm{p})}+\beta_{1}^{(2 \mathrm{p})} q_{1}^{(1 \mathrm{~s})}+\beta_{2}^{(2 \mathrm{p})} q_{2}^{(1 \mathrm{~s})} .
$$

Wir entwickeln nun die Elektronenwellenfunktionen $\psi_{n}$ nach Normalkoordinaten $q_{i}^{(n) 12}$ :

$$
\begin{aligned}
\psi_{n}\left(\mathrm{r}, q_{i}^{(n)}\right) & =\psi_{n}\left(\mathrm{r}, \alpha_{0}^{(n)}+\beta_{1}^{(n)} q_{1}^{(n)}+\beta_{2}^{(n)} q_{2}^{(n)}\right) \\
& =\psi_{n}^{(0)}+\frac{\partial \psi_{n}^{(0)}}{\partial \alpha_{0}^{(n)}}\left[\beta_{1}^{(n)} q_{1}^{(n)}+\beta_{2}^{(n)} q_{2}^{(n)}\right]+\frac{1}{2} \frac{\partial^{2} \psi_{n}^{(0)}}{\partial\left(\alpha_{0}^{(n)}\right)^{2}}\left[\beta_{1}^{(n)} q_{1}^{(n)}+\beta_{2}^{(n)} q_{2}^{(n)}\right]^{2}+\ldots
\end{aligned}
$$

Berücksichtigen wir $\psi_{n}$ bis zu Gliedern erster Ordnung und verwenden die Relation (7), in der die Schwingungskoordinaten $q_{i}^{(2 \mathrm{p})}$ durch die $q_{i}^{(1 \mathrm{~s})}$ ersetzt werden konnten, so lautet das auf Normalkoordinaten transformierte Elektronenintegral:

$\int \psi_{1 \mathrm{~s}}^{*}\left(\mathrm{r}, q_{1}^{(1 \mathrm{~s})}, q_{2}^{(1 \mathrm{~s})}\right) \mathfrak{r} \psi_{2 \mathrm{p}}\left(\mathrm{r}, q_{1}^{(2 \mathrm{p})}, q_{2}^{(2 \mathrm{p})}\right) \mathrm{d}^{3} \mathrm{r}=32\{0,0,1\} \frac{\left(\alpha_{0}^{(1 \mathrm{~s})}\right)^{3 / 2}\left(\alpha_{1 \mathrm{~s}}^{(2 \mathrm{p})}\right)^{5 / 2}}{\left(\alpha_{0}^{(1 \mathrm{~s})}+\alpha_{1 \mathrm{~s}}^{(2 \mathrm{p})}\right)^{5}}\left(1-\delta_{1} q_{1}^{(1 \mathrm{~s})}-\delta_{2} q_{2}^{(1 \mathrm{~s})}\right)$

mit den Abkürzungen

$$
\begin{aligned}
& \delta_{1}=\beta_{1}^{(1 \mathrm{~s})}\left[\frac{5}{\alpha_{0}^{(1 \mathrm{~s})}+\alpha_{1 \mathrm{~s}}^{(2 \mathrm{p})}}-\frac{3}{2 \alpha_{0}^{(1 \mathrm{~s})}}\right]+\beta_{1}^{(2 \mathrm{p})}\left[\frac{5}{\alpha_{0}^{(1 \mathrm{~s})}+\alpha_{1 \mathrm{~s})}^{(2 \mathrm{p})}}-\frac{5}{2 \alpha_{1 \mathrm{~s}}^{(2 \mathrm{p})}}\right] ; \\
& \delta_{2}=\beta_{2}^{(1 \mathrm{~s})}\left[\frac{5}{\alpha_{0}^{(1 \mathrm{~s})}+\alpha_{1 \mathrm{~s}}^{(2 \mathrm{p})}}-\frac{3}{2 \alpha_{0}^{(1 \mathrm{~s})}}\right]+\beta_{2}^{(2 \mathrm{p})}\left[\frac{5}{\alpha_{0}^{(1 \mathrm{~s})}+\alpha_{1 \mathrm{~s}}^{(2 \mathrm{p})}}-\frac{5}{2 \alpha_{1 \mathrm{~s}}^{(2 \mathrm{p})}}\right] .
\end{aligned}
$$

Zur Darstellung der Koeffizienten $\beta_{i}^{(n)}$ mit Hilfe vorgegebener statischer Variationsparameter $\alpha_{0}^{(1 \mathrm{~s})}$ und $\alpha_{0}^{(2 \mathrm{p})}$ verweisen wir ebenso wie für die Berechnung der Oszillatorverschiebungen $a_{i}^{\left(n n^{\prime}\right)}$ auf die Arbeit (I).

Benützt man nur das erste Entwicklungsglied von (8) zur Beschreibung der Dipolmatrixelemente, so treten in (2) Franck-Condon-Integrale der Form ${ }^{13}$

$$
\int \varphi_{m}^{*}(q) \varphi_{m^{\prime}}\left(q^{\prime}\right) \mathrm{d} q^{\prime}=I_{m m^{\prime}}(a)
$$

auf; die Mitnahme des zweiten Termes bedeutet eine Abweichung vom Franck-Condon-Prinzip ${ }^{14}$ und führt auf die Integrale

$$
\int \varphi_{m}^{*}(q) \mathfrak{r} \varphi_{m^{\prime}}\left(q^{\prime}\right) \mathrm{d} q^{\prime}=\frac{x_{0}^{2}}{a}\left[m-m^{\prime}+\frac{a^{2}}{2 x_{0}^{2}}\right] I_{m m^{\prime}}(a)
$$

mit $x_{0}=\sqrt{\hbar / M \overline{\omega_{0}}} ; M$ ist die reduzierte Masse der Gitterkerne, im Falle von $\mathrm{KCl} 31 \cdot 10^{-24} \mathrm{~g}$.

Damit haben wir das Dipolmatrixelement

$$
\begin{aligned}
D_{1 \mathrm{~s} m_{1} m_{2} ; 2 \mathrm{p} m_{1} m_{2^{\prime}}} & =-e \cdot 32 \frac{\left(\alpha_{0}^{(1 \mathrm{~s})}\right)^{\mathrm{s} / 2}\left(\alpha_{1 \mathrm{~s}}^{(2 \mathrm{p})}\right)^{s / 2}}{\left(a_{0}^{(1 \mathrm{~s})}+a_{1 \mathrm{~s}}^{(2 \mathrm{p})}\right)^{5}} I_{m_{1} m_{1^{\prime}}}\left(a_{1}^{(1 \mathrm{~s} 2 \mathrm{p})}\right) \cdot 1_{m_{2} m_{2}}\left(a_{2}^{1 \mathrm{~s} 2 \mathrm{p}}\right) \\
\cdot & \left\{1-\delta_{1} \frac{x_{0}^{2}}{a_{1}^{(1 \mathrm{~s} 2 \mathrm{p})}}\left[m_{1}-m_{1}^{\prime}+\frac{\left(a_{1}^{(1 \mathrm{~s} 2 \mathrm{p})}\right)^{2}}{2 x_{0}^{2}}\right]-\delta_{2} \frac{x_{0}^{2}}{a_{2}^{(1 \mathrm{~s} 2 \mathrm{p})}}\left[m_{2}-m_{2}{ }^{\prime}+\frac{\left(a_{2}^{(1 \mathrm{~s} 2 \mathrm{p})}\right)^{2}}{2 x_{0}^{2}}\right]\right\}
\end{aligned}
$$

explizit vorliegen und kehren zu unserer Ausgangsgleichung (1), der Übergangswahrscheinlichkeit, zurück.

$12 n$ steht für die Zustände $1 \mathrm{~s}$ und $2 \mathrm{p} ; \psi_{n}{ }^{(0)}$ bedeutet $\psi_{n}$ für alle $q_{i}=0(i=1,2)$.

13 S. KoIde, Z. Naturforsch. 15 a, 123 [1960]. - M. WAGNER, Z. Naturforsch. 14 a, 81 [1959] ;

$$
I_{m m^{\prime}}(a)=\exp \left\{-\frac{1}{2} \frac{a^{2}}{2 x_{0}^{2}}\right\}\left(\frac{a}{x_{0} \sqrt{2}}\right)^{m^{\prime}-m} \sqrt{\frac{m !}{m^{\prime} !}} L_{m}^{m^{\prime}-m}\left(\frac{a^{2}}{2 x_{0}^{2}}\right) \text { für } \quad m^{\prime} \geqq m ;
$$

im Falle $m^{\prime}-m \leqq 0$ sind $m$ und $m^{\prime}$ zu vertauschen und $a$ durch $-a$ zu ersetzen; die $L_{m}^{m \prime-m}$ sind Laguerresche Polynome.

14 Siehe Anm. 2, J. J. Markham, S. $362 \mathrm{ff}$. 
Führt man eine mittlere Übergangswahrscheinlichkeit pro Sekunde $p_{v v^{\prime}}$ und eine von der Temperatur $\Theta$ abhängige Besetzungszahl des Ausgangszustandes $N_{v}(\Theta)$ ein und beachtet das Absorptionsgesetz für die Lichtausbreitung in $z$-Richtung

$$
S(\omega, z)=S\left(\omega, z_{0}\right) \exp \left\{-\tau(\omega) \cdot\left(z-z_{0}\right)\right\}
$$

$\left[\tau(\omega)\right.$ ist die Absorptionskonstante, $S(\omega)=\frac{c n}{t}|E(\omega)|^{2}$ die spektrale Lichtintensität (s. Arbeit II) ], so kann die pro Volumeneinheit, Sekunde und Frequenzintervall $\Delta \omega$ absorbierte Energie folgendermaßen angeschrieben werden:

$$
(-\mathrm{d} S(\omega, z) / \mathrm{d} z)_{z=z_{0}} \Delta \omega=S\left(\omega, z_{0}\right) \tau(\omega) \Delta \omega=\underset{\Delta \omega, \hbar \omega=\text { const }}{\hbar} \omega \underset{v}{\infty} N_{v}(\Theta) p_{v v^{\prime}} .
$$

Dabei soll die Summation über sämtliche Übergänge $v \rightarrow v^{\prime}$ ausgeführt werden, deren Energiedifferenz

$$
\hbar \omega_{1 \mathrm{~s} m_{1} m_{2} ; 2 \mathrm{p} m_{1}{ }^{\prime} m_{2}{ }^{\prime}}=E_{0}+\hbar \omega_{0} \mu=\hbar \omega(\mu), \quad \mu=m_{1}{ }^{\prime}-m_{1}+m_{2}{ }^{\prime}-m_{2},
$$

im Intervall $\hbar \omega_{v v^{\prime}} \pm \frac{1}{2} \Delta \omega$ liegt.

Benachbarte Übergänge unterscheiden sich um den Betrag $\hbar \omega_{0}$. Da wir im Intervall $\Delta \omega$ aber $\Delta \omega / \omega_{0}$ solche Übergänge haben, können wir (14) umschreiben:

$$
\tau(\omega(\mu))=\frac{4 \pi^{2}}{\hbar n c} \frac{\hbar \omega(\mu)}{\hbar \omega_{0}} \sum_{\mu=\mathrm{const}} N_{1 \mathrm{~s} m_{1} m_{2}}(\Theta)\left|D_{1 \mathrm{~s} m_{1} m_{2} ; 2 \mathrm{p} m_{1^{\prime} m_{2}}}\right|^{2} .
$$

Die Summation erstreckt sich über sämtliche Kombinationen der Quantenzahlen $m_{i}$ und $m_{i}{ }^{\prime}$, für die $\mu$ konstant bleibt.

Wir vernachlässigen die Temperaturabhängigkeit der Besetzungszahl und setzen die Zahl der im 1s-Zustand besetzten F-Zentren pro $\mathrm{cm}^{3} N_{1 \mathrm{~s}} \approx N_{\mathrm{F}}$, der Gesamtzahl der pro $\mathrm{cm}^{3}$ vorhandenen F-Zentren; dazu kommt noch die Annahme, daß sich die Oszillatoren vor dem Elektronenübergang im thermischen Gleichgewicht befinden.

$$
N_{1 \mathrm{~s} m_{1} m_{2}}(\Theta)=N_{1 \mathrm{~s}} W_{m_{1}}(\Theta) W_{m_{\mathrm{q}}}(\Theta)=N_{1 \mathrm{~s}}(1-\lambda)^{2} \lambda^{m_{1}+m_{2}}:
$$

mit dem Boltzmann-Faktor $k$ und $\lambda=\exp \left\{-\hbar \omega_{0} / k \Theta\right\}$; dabei ist $W_{m}(\Theta)$ die Wahrscheinlichkeit dafür, da $ß$ der Oszillator bei einer Temperatur $\Theta$ einen Schwingungszustand $m$ hat.

In (16) läßt sich die Summation auf der rechten Seite geschlossen ausführen ${ }^{15}$, und man bekommt mit Hilfe von Gl. (12) und Gl. (17) :

$$
\begin{aligned}
\tau(\omega(\mu), \Theta) & =K \frac{\hbar \omega(\mu)}{\hbar \omega_{0}}\left[1-2\left(1-\frac{|\mu|}{x+y}\right)\left(\delta_{1}^{\prime} x+\delta_{2}{ }^{\prime} y\right)\right] \cdot \exp \left\{-\frac{1+\lambda}{1-\lambda}(x+y)+\frac{\hbar \omega_{0}|\mu|}{2 k \Theta}\right\} \cdot I_{\mu}(\bar{z}) ; \quad(18) \\
\operatorname{mit} \quad K & =\frac{4 \pi^{2} e^{2}}{\hbar n c} N_{\mathrm{F}}\left[32 \frac{\left(\alpha_{0}^{(1 \mathrm{~s})}\right)^{3 / 2}\left(\alpha_{1 \mathrm{~s}}^{(2 \mathrm{p})}\right)^{5 / 2}}{\left(\alpha_{0}^{(1 \mathrm{~s})}+\alpha_{1 \mathrm{~s}}^{(2 \mathrm{p})}\right)^{5}}\right]^{2}, \\
\bar{z} & =\frac{2(x+y) \lambda}{1-\lambda}, \quad x=\frac{\left(a_{1}^{(1 \mathrm{~s} 2 \mathrm{p})}\right)^{2}}{2 x_{0}^{2}}, \quad y=\frac{\left(a_{2}^{(1 \mathrm{~s} 2 \mathrm{p})}\right)^{2}}{2 x_{0}^{2}}, \quad \delta_{1}{ }^{\prime}=\delta_{1} \frac{x_{0}^{2}}{a_{1}^{(1 \mathrm{~s} 2 \mathrm{p})}}, \quad \delta_{2}{ }^{\prime}=\delta_{2} \frac{x_{0}^{2}}{a_{2}^{(1 \mathrm{~s} 2 \mathrm{p})}}
\end{aligned}
$$

und der modifizierten Bessel-Funktion

$$
I_{\mu}(\bar{z})=\left(\frac{1}{2} \bar{z}\right)^{\mu} \sum_{r=0}^{\infty}\left(\frac{1}{2} \bar{z}\right)^{2 r} \frac{1}{r !(r+\mu) !} .
$$

Den Exponentialfaktor in (18) kürzen wir mit $E(\mu, \Theta)$ ab und den Ausdruck in der eckigen Klammer mit $C(\mu)$ und berechnen die Absorption

$$
\bar{\tau}=\tau / K=\left[\omega(\mu) / \omega_{0}\right] \cdot C(\mu) \cdot E(\mu, \Theta) \cdot I_{\mu}(\bar{z}(\Theta))
$$

mit variablem Temperaturparameter in Abhängigkeit von $\mu$ (s. Abb. 1), das wir in Einheiten von $\Delta \omega$ auftragen.

15 Siehe (II), mathematischer Anhang; dabei ist zu beachten, daß in (A 4) die obige Aussage sowohl für $\mu \geqq 0$ als auch

für $\mu \leqq 0$ Gültigkeit hat, wenn sämtliche $\mu$ durch $|\mu|$ ersetzt werden. 
Das Maximum der Absorptionsbande, dessen Lage unabhängig von der Wahl der in (15) eingeführten Energiekonstanten $E_{0}$ ist, zeigt zwischen $\Theta=20^{\circ} \mathrm{K}$ und $\Theta=320^{\circ} \mathrm{K}$ einen kaum merklichen Temperaturgang von ungefähr $0,01 \mathrm{eV}$ und überdies mit steigender Temperatur zu höheren Energiewerten hin, während das Experiment ${ }^{2}$ in diesem $\Theta$-Bereich eine Verschiebung von $0,1 \mathrm{eV}$ in Richtung fallender Energie liefert.

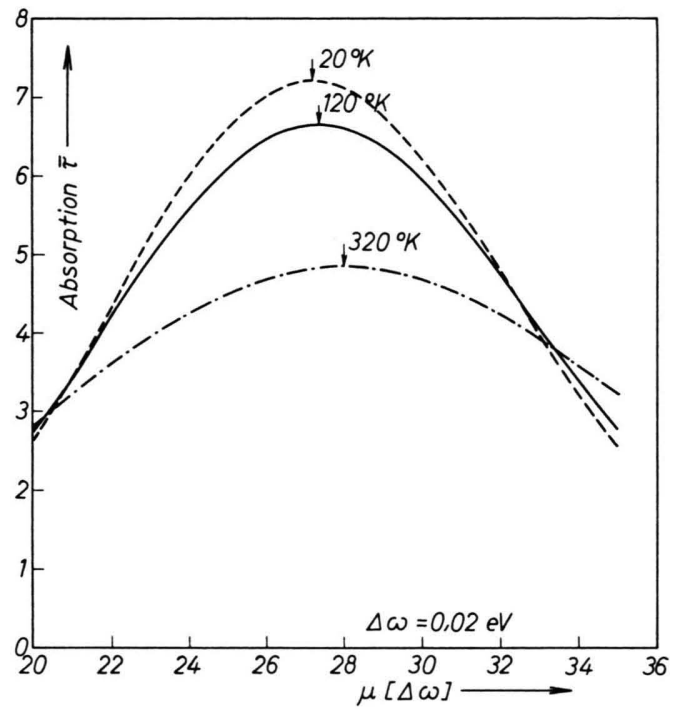

Abb. 1. Die F-Bande von $\mathrm{KCl}$ bei 3 verschiedenen Temperaturen für den Fall frequenzunabhängiger Franck-Condon-Integrale. Es wurden folgende Daten verwendet: $E_{0}=1,76 \mathrm{eV}$; $\alpha_{1 \mathrm{~s}}=5,5 \cdot 10^{7} \mathrm{~cm}^{-1} ; \alpha_{2 \mathrm{p}}=0,4 \cdot 10^{7} \mathrm{~cm}^{-1}$.

Unser $\bar{\tau}$ unterscheidet sich von der WAGNerschen Rechnung ${ }^{16}$ um den Faktor $C(\mu)$, der aber, wie sich jetzt herausstellt, den Temperatureffekt nicht erklärt. In der Arbeit (II) von WagNer wurde also zu Recht $C(\mu)=1$ gesetzt. Eine genaue Nachrechnung der dort aufgezeichneten Kurve $\bar{\tau}^{\prime}$ ergibt, daß sie schon mit der nullten Näherung aus Gl. (3) die Tendenz eines Temperaturganges in verkehrter Richtung aufweist. Die Verschiebung von

$$
\bar{\tau}^{\prime}=\frac{\omega(\mu)}{\omega_{0}} \cdot 1 \cdot E(\mu, \Theta) \cdot I_{\mu}(\bar{z}(\Theta))
$$

ist allerdings noch um den Faktor $\frac{1}{2}$ kleiner als in dem von uns behandelten Fall.

\section{§ 2. Änderung der Oszillatorfrequenz beim Elektronenübergang}

In unserem zweiten Modell setzen wir wieder voraus, daß mit dem Übergang des Störstellenelektrons in ein höheres Niveau nur 2 Gitterschwingungen korreliert sind. Zu beiden Gitteroszillatoren gehören im ls-Zustand die Ruhelagen $q_{i}$ und die gleichen Frequenzen $\omega_{0}$, im 2p-Zustand $q_{i}^{\prime}$ und gleiche $\omega^{\prime}$.

$$
\omega^{\prime}=\omega_{0}-\Omega \text {. }
$$

Die Diskussion am Ende von $\S 1$ erbrachte, daß es bei Berechnung des Dipolmatrixelements

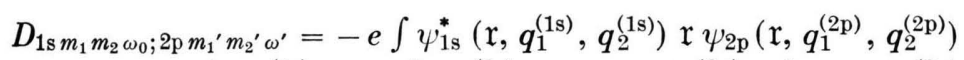

$$
\cdot \varphi_{m_{1}}^{*}\left(q_{1}^{(1 \mathrm{~s})}, \omega_{0}\right) \varphi_{m_{2}}^{*}\left(q_{2}^{(1 \mathrm{~s})}, \omega_{0}\right) \cdot \varphi_{m_{1}}\left(q_{1}^{(2 \mathrm{p})}, \omega^{\prime}\right) \cdot \varphi_{m_{2}}\left(q_{2}^{(2 \mathrm{p})}, \omega^{\prime}\right) \mathrm{d}^{3} \mathfrak{r} \mathrm{d} q_{1}^{(1 \mathrm{~s})} \mathrm{d} q_{2}^{(1 \mathrm{~s})}
$$

genügt, Gl. (8) nach dem Entwicklungsglied nullter Ordnung der Elektronenwellenfunktion abzubrechen. Man kommt jetzt bei der Berechnung der Absorptionskonstanten für die Franck-Condon-Integrale auf Ausdrücke mit Hermiteschen Polynomen $\mathrm{He}_{m}{ }^{17}$ :

16 Siehe (II), Abb. 2, S. 312.

17 Siehe Anm. ${ }^{13}$; man beachte, daß die Oszillatorenverschiebungen $a$ bei WAGNER und $\Delta q$ bei KoIDE auf Grund der je- weils angesetzten Hamilton-Operatoren folgenden Zusammenhang haben: $\Delta q=a \sqrt{M}$. 


$$
|\boldsymbol{J}|^{2}=\left.\int_{-\infty}^{+\infty} \varphi_{m}\left(q^{\prime}-a, \omega_{0}\right) \varphi_{m^{\prime}}\left(q^{\prime}, \omega^{\prime}\right) \mathrm{d} q^{\prime}\right|^{2}=\frac{\exp \left\{-\alpha^{2}\right\}}{m ! m^{\prime} !} \frac{2 \sqrt{\omega_{0} \omega^{\prime}}}{\omega_{0}+\omega^{\prime}}\left(\frac{\omega_{0}-\omega^{\prime}}{\omega_{0}+\omega^{\prime}}\right)^{m^{\prime}}\left|D^{m} F\right|^{2},
$$

wobei

$$
\begin{aligned}
D^{m} F & =\sum_{k=0}^{m}\left(\begin{array}{c}
m \\
k
\end{array}\right)(-\alpha)^{m-k} \frac{\partial^{k}}{\partial \alpha^{k}} \operatorname{He}_{m}(\alpha \xi) \exp \left\{\frac{\omega_{0}-\omega^{\prime}}{\omega_{0}+\omega^{\prime}} \frac{\alpha^{2}}{2}\right\}, \\
\alpha^{2} & =a^{2} \frac{M \omega_{0}}{2 \hbar}, \quad \xi=\left\{\begin{array}{lll}
\sqrt{4 \omega_{0} \omega^{\prime} /\left(\omega_{0}^{2}-\omega^{\prime 2}\right)} & \text { für } & \omega_{0}>\omega^{\prime}, \\
\left.i \sqrt{4 \omega_{0} \omega^{\prime} /\left(\omega^{\prime 2}-\omega_{0}^{2}\right.}\right) & \text { für } & \omega_{0}<\omega^{\prime}
\end{array}\right.
\end{aligned}
$$

und ${ }^{18}$

$$
\mathrm{He}_{m}(z)=m ! \sum_{r=0}^{[m / 2]} \frac{(-1)^{r} z^{m-2 r}}{r ! 2^{r}(m-2 r) !}:
$$

[ $m / 2]$ bedeutet die größte ganze Zahl $\leqq m / 2$.

Gl. (23) läßt sich nach einigen Umrechnungen und mit Hilfe der Rodrigues-Formeln ${ }^{19}$ auf eine für die numerische Rechnung brauchbarere Form bringen:

$$
\begin{aligned}
&|\mathcal{J}|^{2}=\frac{2}{m ! m^{\prime} !} \exp \left\{-\alpha^{2}(1-B)\right\} \bar{F} B^{m^{\prime}} \cdot \mid \sum_{\gamma=0}^{m}\left(\begin{array}{c}
m \\
\gamma
\end{array}\right)(-\alpha)^{m-\gamma} \\
&\left.\cdot \sum_{\nu=0}^{\min \left(\gamma_{\gamma} m^{\prime}\right)} v !\left(\begin{array}{c}
\gamma \\
v
\end{array}\right)\left(\begin{array}{c}
m^{\prime} \\
v
\end{array}\right)(i \sqrt{B})^{\gamma-\nu} \xi^{\nu}(-1)^{\nu-\gamma} \mathrm{He}_{m^{\prime}-\nu}(\alpha \xi) \cdot \mathrm{He}_{\gamma-\nu}(i \sqrt{B} \alpha)\right|^{2} .
\end{aligned}
$$

Die Abkürzungen $B, \xi$ und $\bar{F}$ haben folgende Gestalt:

$$
\begin{aligned}
& B=\Omega /\left(2 \omega_{0}-\Omega\right) ; \quad \bar{F}=\sqrt{\omega_{0}\left(\omega_{0}-\Omega\right)} /\left(2 \omega_{0}-\Omega\right) ; \\
& \xi= \begin{cases}2 \sqrt{\omega_{0}\left(\omega_{0}-\Omega\right) /\left(2 \omega_{0}-\Omega\right) \Omega} & \text { für } \Omega>0, \\
2 i \sqrt{\omega_{0}\left(\omega_{0}-\Omega\right) /\left(2 \omega_{0}-\Omega\right)(-\Omega)} & \text { für } \Omega<0 .\end{cases}
\end{aligned}
$$

Wir verwenden die gleichen Wasserstoffunktionen (4) wie WAGNER; somit ist mit (5) und (24) das Dipolmatrixelement für unser Modell bekannt. Die Überlegungen, die zu den Gln. (13), (14) und (17) führten, haben weiterhin Gültigkeit, nur daß wir jetzt Übergänge mit anderer Energiedifferenz betrachten:

$$
\hbar \omega_{1 \mathrm{~s} m_{1} m_{2} ; 2 \mathrm{p} m_{1}{ }^{\prime} m_{2}{ }^{\prime}}=E_{0}+\hbar\left(\omega^{\prime} m_{1}^{\prime}-\omega_{0} m_{1}+\omega^{\prime} m_{2}{ }^{\prime}-\omega_{0} m_{2}\right)=E_{0}+\hbar\left(\omega_{0} \mu-\Omega \mu^{\prime}\right),
$$

wo $\mu$ [s. Gl. (15)] wieder die Summe der Quantenzahldifferenzen ist und $\mu^{\prime}=m_{1}{ }^{\prime}+m_{2}{ }^{\prime}$.

Die geschlossene Aufsummation über alle Übergänge, die in ein Energieintervall $\Delta \omega$ fallen, gelingt dieses Mal nicht mehr. Wir führen daher analog zu (15) ein $\omega(\varkappa)$ mit einer diskreten Veränderlichen $\varkappa$ ein; diesem $\omega(\varkappa)$ ordnen wir alle möglichen Energiedifferenzen zu, die in den Bereich $(\varkappa-1) \Delta \omega<\omega_{0} \mu$ $-\Omega \mu^{\prime} \leqq \varkappa \Delta \omega$ fallen. Somit haben wir für die Absorptionskonstante an der Stelle $\varkappa$ folgende Formel auszuwerten:

$$
\hat{\tau}=\frac{\tau(\omega(\varkappa), \Theta)}{K}=\frac{\omega(\varkappa)}{\Delta \omega}(1-\lambda)^{2} \cdot \sum_{(x-1)<\frac{\omega_{0} \mu-\Omega \mu^{\prime}}{\Delta \omega} \leq \varkappa} \lambda_{m_{1}+m_{2}}\left|J\left(m_{1}, m_{1}{ }^{\prime}\right)\right|^{2} \mid J\left(m_{2}, m_{2}{ }^{\prime}\right)^{2} .
$$

In (26) finden die gleichen Bezeichnungen wie in (24) Verwendung, wobei $\mathcal{J}\left(m_{i}, m_{i}^{\prime}\right)$ bedeutet, da $m$ und $m^{\prime}$ durch die Quantenzahlen $m_{i}$ und $m_{i}^{\prime}(i=1,2)$ ersetzt werden; an die Stelle von $\alpha$ in Gl. (24) treten jetzt die Symbole $\alpha_{i}$, die mit den Oszillatorverschiebungen $a_{i}$ folgendermaßen verknüpft sind:

$$
\alpha_{i}^{2}=a_{i}^{2} \frac{M \omega_{0}}{2 \hbar} \quad(i=1,2) .
$$

Für die Temperaturen $20^{\circ} \mathrm{K}$ und $320^{\circ} \mathrm{K}$ ist in Abb. 2 die Absorption $\hat{\tau}$ gegen $\varkappa$ (wie $\mu$ wird $\varkappa$ in Maßeinheiten von $\Delta \omega$ aufgetragen) bei drei verschiedenen Werten $\omega^{\prime}$ aufgezeichnet. Wir nehmen bei unseren

19 Siehe Anm. ${ }^{18}$, S. 252.

18 Zur Summendarstellung der Hermiteschen Polynome siehe W. Magnus, F. Oberhettinger und R. P. Soni, Formulas and
Theorems of the Special Functions of Mathematical Physics, Springer-Verlag, Berlin 1966, S. 250. 
Rechnungen alle Übergänge $v \rightarrow v^{\prime}$ mit, für die die Summe der Oszillatorquantenzahlen im Anfangszustand 10 ist (d.h. $\left.m_{1}+m_{2}=10\right)$ und die Summe der $m_{i}{ }^{\prime} 50$ beträgt $\left(m_{1}{ }^{\prime}+m_{2}{ }^{\prime}=50\right)$; dabei treten in Gl. (26) in jedem Energieintervall $(\varkappa-1) \Delta \omega<\omega_{0} \mu-\Omega \mu^{\prime} \leqq \varkappa \Delta \omega$, mit $\Delta \omega$ ungefähr $0,02 \mathrm{eV}$, bis zu 3000 Summenglieder auf. Die entscheidenden Beiträge, die die Größenordnung der Absorption festlegen, liefern aber nur die Fälle, bei denen sich beide ankoppelnden Oszillatoren in den Anfangszuständen $m_{1}=m_{2}=0$ befinden. Um zu vermeiden, daß sich Schwankungen in der Verteilungskurve ergeben, die nicht dem physikalischen Sachverhalt entsprechen, müssen wir die Gewähr haben, daß jedem $\omega(\varkappa)$ genau eine Schwingungskonstellation $m_{1}+m_{2}=0$ zugeordnet ist; deshalb wählen wir bei allen Kurvenpaaren als Abszisse einen anderen Energiemaßstab, nämlich stets $\Delta \omega=\omega^{\prime}$.

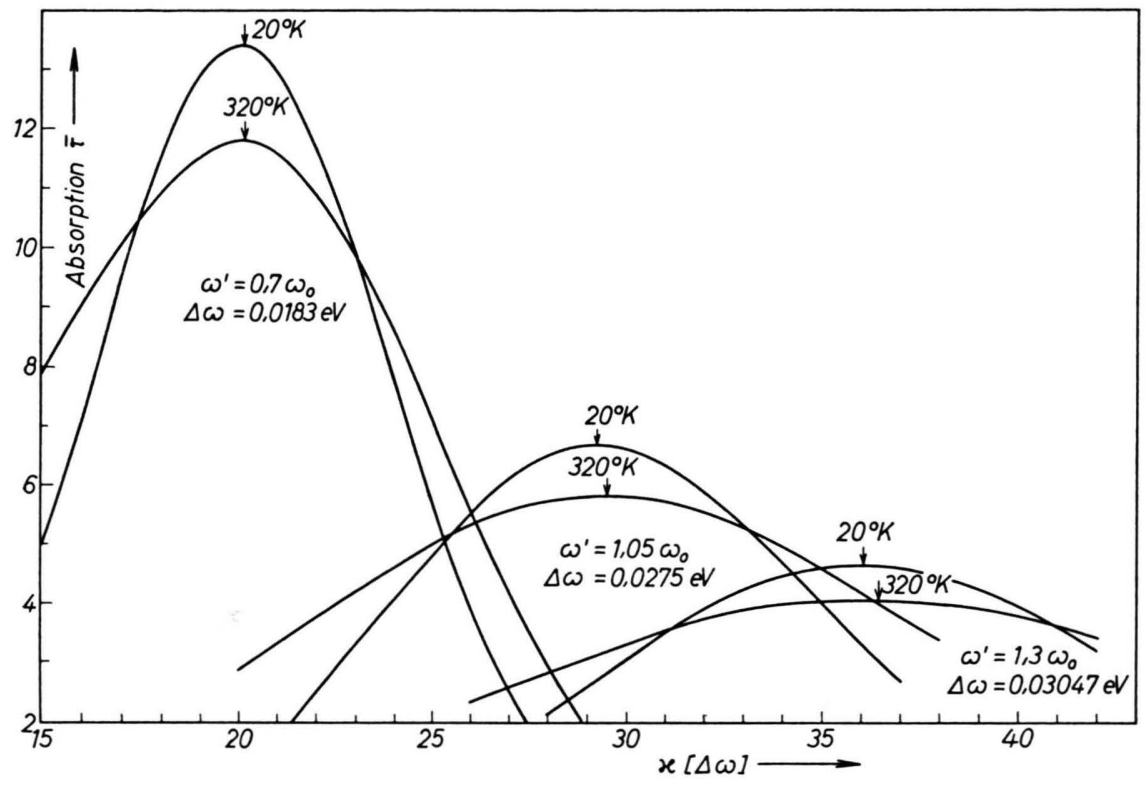

Abb. 2. Drei Beispiele der FBande für den Fall $\omega_{0} \neq \omega^{\prime}$ [Anm.: Für die numerischen Parameter wurden die gleichen Daten wie in $\$ 1$ (s. Abb. 1) eingesetzt; außerdem bedeutet das hier aufgezeichnete $\bar{\tau}$ die Absorption $\hat{\tau}$ aus Gl. (26) ].

Wir sehen, daß sich auch mit dem Modell der geänderten Oszillatorfrequenzen der Temperaturgang der F-Absorptionsbande nicht erklären läßt; denn das Maximum zeigt bei verschiedenen Temperaturen fast keine Verschiebung. Man findet wieder, in der gleichen Größenordnung wie bei den Rechnungen nullter Näherung in $\S 1$, eine Verlagerung der Bande in die vom Experiment her nicht erwartete Richtung.

\section{§ 3. Vergleich der Modelle mit den Arbeiten von Dexter und Pryce}

Wir haben in unseren Ausführungen vergeblich versucht, die Temperaturabhängigkeit der Lage der F-Bande unter zwei verschiedenen Gesichtspunkten, beidesmal im Rahmen der adiabatischen Näherung ${ }^{20}$, zu klären: Einmal nahmen wir eine Änderung der statischen Verhältnisse des Kristalls beim Ưbergang des gebundenen Elektrons in einen angeregten $\mathrm{Zu}$ -

20 M. Born u. K. Huang, Dynamical Theory of Crystal Lattices, Clarendon Press, Oxford 1954, S. 170. - H. Stumpf, Quantentheorie der Ionenrealkristalle, Springer-Verlag, Berlin 1961, S. 14 ff., S. 110 ff. - J. M. Ziman, Electrons and Phonons, Clarendon Press, Oxford 1960, S. $172 \mathrm{ff}$. stand an und zum anderen eine Änderung im dynamischen Verhalten.

Unsere an WAGNER orientierten Rechnungen in $\S 1$ beinhalten eine Beeinflussung der statischen Eigenschaften des Kristalls beim Elektronenübergang, wie sich bei der Berechnung der Gitterenergie zeigt $^{21}$, da die höheren Entwicklungsglieder der Elektronenwellenfunktion nicht nur eine Abweichung vom Franck-Condon-Prinzip ${ }^{22}$ erzwingen, sondern

21 Siehe Anm. ${ }^{6}$, (I), Gl. (63) .

22 Siehe Anm. ${ }^{14}$. 
auch die potentielle Energie um frequenzändernde Terme erweitern.

Beim zweiten Modell haben wir im Konfigurationskoordinatendiagramm nur „senkrechte Übergänge" berücksichtigt ${ }^{23}$. Wir bleiben also innerhalb der Franck-Condon-Vorstellung, da wir aus $\S 1$ wissen, daß Abweichungen davon vernachlässigt werden können; dafür rechnen wir aber im angeregten $\mathrm{Zu}$ stand mit einer anderen Parabelöffnung als im Grundzustand, d. h. mit einem anderen dynamischen Verhalten.

Die gegen Temperatureinflüsse invariante Lage unserer Bande scheint den Ergebnissen von Dexter ${ }^{24}$ und Pryce ${ }^{25}$ zu widersprechen. Dexter hat, aufbauend auf Arbeiten von O'Rourke ${ }^{26}$ und Lax ${ }^{27}$, die Absorptionsbande für ein dem unseren entsprechendes Modell aus $N$ harmonischen Oszillatoren gleicher Frequenz formal angegeben. Die Oszillatoren haben bei verschiedenen Elektronenzuständen verschiedene Schwingungszahlen. Der Wechselwirkung zwischen einem Störzentrum beliebiger Art und der Gitterbewegung wird durch einen linearen Wechselwirkungsoperator Rechnung getragen. Für das Spektrum hat Dexter durch die Berechnung des 1. Moments die Temperaturabhängigkeit des Mittelwertes der Bande angeschrieben, bei der weiteren Diskussion aber keine Folgerungen daraus gezogen. Ebenfalls mit der Momentenmethode, also auf O'Rourke und Lax fußend, konnte Pryce den Temperaturgang des Mittelwertes auf eine sehr elegante Art aufzeigen; es gelingt ihm nämlich, ohne Kenntnis der Bandenfunktion, das nach Einführung dynamisch veränderlicher Kopplungskonstanten von Null verschiedene Moment erster Ordnung anzuschreiben. Nach Pryce, der somit auch wie wir in $\S 2$ ein tem-

23 Siehe Anm. 2, J. J. Markham, S. 353 ff. u. S. 367.

24 D. L. Dexter, Sol. State Phys. 6, 353 [1958].

25 M. H. L. Pryce, Phonons in Perfect Lattices and in Lattices with Point Imperfections, Oliver \& Boyd, Edinburgh and London 1965. peraturabhängiges dynamisches Verhalten annimmt, ergibt sich klar, daß der Mittelwert mit steigender Temperatur in die Richtung niedrigerer Energien verlagert wird; allerdings erfolgt keine Aussage über die Größe des Effekts.

Die Ergebnisse von Dexter und Pryce, die das gleiche Modell wie wir in $\S 2$ verwenden, beziehen sich auf einen Temperaturgang der Mittelwerte; wir dagegen haben die gesamte Bande berechnet, wobei sich praktisch keine Maximumverschiebung ergeben hat. Nun gilt zu bedenken, daß Maximum und Mittelwert nur bei einer symmetrischen Verteilungsfunktion notwendig zusammenfallen und gleiche funktionelle Abhängigkeit zeigen müssen, nicht aber bei unsymmetrischen Kurven. Man weiß, daß bei der F-Bande in der Tat eine von der Temperatur beeinflußte Asymmetrie existiert ${ }^{28}$, die wir mit unserer Theorie auch recht gut erfassen ${ }^{29}$.

Abschließend können wir sagen, daß der Temperaturgang der Mittelwerte der F-Absorptionsbande vor allem durch den Asymmetrieeffekt bedingt ist und daß, wie unsere Rechnungen deutlich zeigen, es mit den von uns behandelten Modellen nicht möglich ist, die Maximumsverschiebungen zu erklären.

Ich danke Herrn Prof. Dr. H. Stumpf, der das Thema anregte, und Herrn Prof. Dr. F. Bopp, an dessen Lehrstuhl ich die Arbeit ausführen durfte. Besonders bedanke ich mich bei den Herren Dr. F. WAHL, Dr. H. Maier-Bötzel und D. Stocker für wertvolle Hinweise. Mein Dank gilt auch der Deutschen Forschungsgemeinschaft für finanzielle Förderung und dem LeibnizRechenzentrum der Bayerischen Akademie der Wissenschaften, auf deren Großrechenanlage TR 4 ich die sehr umfangreichen numerischen Rechnungen ausführen konnte.

26 R. C. O'Rourke, Phys. Rev. 91, 265 [1953].

27 M. LAX, J. Chem. Phys. 20, 1752 [1952].

28 Siehe Anm. ${ }^{2}$.

29 Siehe (II), § 3 . 